TRANSACTIONS OF THE

AMERICAN MATHEMATICAL SOCIETY

Volume 349, Number 3, March 1997, Pages 1081-1089

S 0002-9947(97)01902-8

\title{
SIGNED QUASI-MEASURES
}

\author{
D. J. GRUBB
}

\begin{abstract}
Let $X$ be a compact Hausdorff space and let $\mathcal{A}$ denote the subsets of $X$ which are either open or closed. A quasi-linear functional is a map $\rho: C(X) \rightarrow \mathbf{R}$ which is linear on singly generated subalgebras and such that $|\rho(f)| \leq M\|f\|$ for some $M<\infty$. There is a one-to-one correspondence between the quasi-linear functional on $C(X)$ and the set functions $\mu: \mathcal{A} \rightarrow \mathbf{R}$ such that i) $\mu(\emptyset)=0$, ii) If $A, B, A \cup B \in \mathcal{A}$ with $A$ and $B$ disjoint, then $\mu(A \cup B)=\mu(A)+\mu(B)$, iii) There is an $M<\infty$ such that whenever $\left\{U_{\alpha}\right\}$ are disjoint open sets, $\sum\left|\mu\left(U_{\alpha}\right)\right| \leq M$, and iv) if $U$ is open and $\varepsilon>0$, there is a compact $K \subseteq U$ such that whenever $V \subseteq U \backslash K$ is open, then $|\mu(V)|<\varepsilon$. The space of quasi-linear functionals is investigated and quasi-linear maps between two $C(X)$ spaces are studied.
\end{abstract}

Let $X$ be a compact Hausdorff space and $C(X)$ the space of real-valued continuous functions on $X$. A map $\rho: C(X) \rightarrow \mathbf{R}$ is said to be a quasi-linear functional if $\rho$ is linear on singly generated subalgebras and bounded in the sense that there exists an $M<\infty$ such that $|\rho(f)| \leq M\|f\|_{u}$ for all $f \in C(X)$. Let $\|\rho\|$ be the minimal such $M$. If $\rho$ and $\eta$ are quasi-linear functionals, we define $\rho+\eta$ by pointwise action on functions. In this fashion, the collection of all quasi-linear functionals becomes a normed linear space. Call this space $Q L(X)$.

Notice that if $\rho$ is quasi-linear, and $f g=0$, then $\rho(f+g)=\rho(f)+\rho(g)$. In fact, if $f$ and $g$ are also positive, we have that the subalgebra generated by $f-g$ contains both $f$ and $g$. In general, we can break $f$ and $g$ into positive and negative parts to get the result. Also notice that if $c$ is a constant, $\rho(c+f)=\rho(c)+\rho(f)$. Thus, if $f$ is constant on the support of $g$, we still have that $\rho(f+g)=\rho(f)+\rho(g)$.

Our goal is to find set functions that produce all quasi-linear functionals on $C(X)$. We will use an approach inspired by the techniques in [1] where the theory of positive quasi-linear functionals is presented. We use the notation $f \prec U$ when $U$ is open to state that $0 \leq f \leq 1$ and $f$ has support contained in $U$. We also use the notation sp $f$ for the image of $f$.

Let $\mathcal{O}$ be the collection of open sets in $X$ and $\mathcal{C}$ the collection of closed sets. Also, let $\mathcal{A}=\mathcal{O} \cup \mathcal{C}$. Thus $\mathcal{A}$ is the collection of subsets of $X$ which are either open or closed.

Definition 1. A function $\mu: \mathcal{A} \rightarrow \mathbf{R}$ is called a signed quasi-measure if the following hold:

(i) $\mu(\emptyset)=0$,

(ii) If $A, B \in \mathcal{A}$ are disjoint with $A \cup B \in \mathcal{A}$, then $\mu(A \cup B)=\mu(A)+\mu(B)$,

Received by the editors August 20, 1995.

1991 Mathematics Subject Classification. Primary 28C05.

(C)1997 American Mathematical Society 
(iii) There is a constant $M<\infty$ such that whenever $\left\{U_{n}\right\}$ is a finite disjoint collection of open sets, then $\sum\left|\mu\left(U_{n}\right)\right| \leq M$,

(iv) If an open set $U$ and $\varepsilon>0$ are given, there exists a closed set $K \subseteq U$ such that if $V$ is an open set with $V \subseteq U \backslash K$, we have $|\mu(V)|<\varepsilon$.

We define $\|\mu\|$ to be the minimal $M$ such that (iii) holds.

For future reference, we note that property (ii) above is equivalent to the following three statements.

a) If $U$ and $V$ are disjoint open sets, then $\mu(U \cup V)=\mu(U)+\mu(V)$.

b) If $U$ and $V$ are open with $X=U \cup V$, then $\mu(U)+\mu(V)=\mu(X)+\mu(U \cap V)$.

c) If $U$ is open, then $\mu(X \backslash U)=\mu(X)-\mu(U)$.

This will allow us to define a quasi-measure by its action on only open sets.

Let $Q M(X)$ denote the collection of all signed quasi-measures on $X$. If we define $\mu+\nu$ by action on sets, we see that $Q M(X)$ is a normed linear space. We wish to show that in a natural way $Q L(X)$ and $Q M(X)$ are isomorphic as normed linear spaces, and are, in fact, Banach spaces.

Given a signed quasi-measure $\mu$, we may define a new set function $|\mu|$ on open sets by

$$
|\mu|(U)=\sup \left\{\sum\left|\mu\left(U_{n}\right)\right|: U_{n} \subseteq U \text { are disjoint open sets }\right\} .
$$

Then we see that $\|\mu\|=|\mu|(X)$. It is important to note here that $|\mu|$ need not yield a quasi-measure. In particular, it is impossible to define $|\mu|$ on closed sets so that (ii) holds. An example of this will be seen later. It is clear, however, that (i) and (iii) hold for $|\mu|$. We will see later that (iv) does also.

Proposition 2. We have the following:

a) If $\left\{A_{\alpha}\right\}_{\alpha \in A} \subseteq \mathcal{A}$ is a collection (possibly infinite) of disjoint subsets of $U \in \mathcal{O}$, then $\sum\left|\mu\left(A_{\alpha}\right)\right| \leq|\mu|(U)$.

b) If $U_{1} \subseteq U_{2} \subseteq \cdots$ are open, then $\mu\left(\bigcup_{i=1}^{\infty} U_{i}\right)=\lim _{i \rightarrow \infty} \mu\left(U_{i}\right)$.

c) If $\left\{U_{\alpha}\right\}$ is a collection (possibly infinite) of disjoint open sets, then $\mu\left(\bigcup U_{\alpha}\right)=$ $\sum \mu\left(U_{\alpha}\right)$.

Proof. Both b) and c) are results of the regularity assumption (iv). For a), notice that there is a similar outer regularity for closed sets, so if $\left\{F_{n}\right\}_{n=1}^{N}$ are finitely many closed sets contained in $U$, and $\varepsilon>0$, we may find disjoint open sets $U_{n}$ with $F_{n} \subseteq$ $U_{n} \subseteq U$ and $\left|\mu\left(U_{n} \backslash F_{n}\right)\right|<\varepsilon / N$. Then $\sum\left|\mu\left(F_{n}\right)\right| \leq \sum\left|\mu\left(U_{n}\right)\right|+\varepsilon \leq|\mu|(U)+\varepsilon$. For the general case, we may restrict to finitely many $A_{\alpha}$, and approximate by closed sets using inner regularity for open sets.

Now, let $f \in C(X)$ and $\alpha \in \mathbf{R}$, and define $\check{f}(\alpha)=\mu\left(f^{-1}(\alpha,+\infty)\right)$ and $\hat{f}(\alpha)=$ $\mu\left(f^{-1}[\alpha,+\infty)\right)$. Notice that $\mu\left(f^{-1}(\alpha, \beta)\right)=\check{f}(\alpha)-\hat{f}(\beta)$ and $\mu\left(f^{-1}[\alpha, \beta]\right)=\hat{f}(\alpha)-$ $\check{f}(\beta)$.

Proposition 3. Let $f \in C(X)$. Then

a) $\check{f}$ is continuous from the right and $\hat{f}$ is continuous from the left.

b) $\check{f}\left(\alpha^{-}\right)=\hat{f}(\alpha)$ and $\hat{f}\left(\alpha^{+}\right)=\check{f}(\alpha)$.

c) $\check{f}$ and $\hat{f}$ agree except at countably many $\alpha \in \mathbf{R}$.

d) If $\check{f}(\alpha)=\hat{f}(\alpha)$, then $\check{f}$ is continuous at $\alpha$.

e) $\check{f}$ is of bounded variation with variation less than $\|\mu\|$. 
Proof. a) If $\alpha_{n}$ decreases to $\alpha$, then

$$
\begin{aligned}
\check{f}(\alpha) & =\mu\left(f^{-1}(\alpha,+\infty)\right) \\
& =\lim _{n \rightarrow \infty} \mu\left(f^{-1}\left(\alpha_{n},+\infty\right)\right) \\
& =\lim _{n \rightarrow \infty} \check{f}\left(\alpha_{n}\right) .
\end{aligned}
$$

Thus $\check{f}$ is continuous from the right. Since $\hat{f}(\alpha)=\mu(X)-(-f)^{\vee}(-\alpha), \hat{f}$ is continuous from the left.

b) Let $\alpha_{0}$ and $\varepsilon>0$ be given and let $U=f^{-1}\left(\alpha_{0},+\infty\right)$. Pick $K$ as in iv) of the definition of quasi-measure. Let $\beta$ be the minimum value of $f$ on $K$. Then $\alpha_{0}<\beta$. If $\alpha_{0}<\alpha<\beta$, we have that $f^{-1}\left(\alpha_{0}, \alpha\right) \subseteq U \backslash K$, so $\left|\check{f}\left(\alpha_{0}\right)-\hat{f}(\alpha)\right|=\left|\mu\left(f^{-1}\left(\alpha_{0}, \alpha\right)\right)\right|<\varepsilon$. This shows that $\hat{f}\left(\alpha_{0}^{+}\right)=\check{f}\left(\alpha_{0}\right)$.

c) Notice that $\sum|\hat{f}(\alpha)-\check{f}(\alpha)|=\sum\left|\mu\left(f^{-1}(\{\alpha\})\right)\right| \leq\|\mu\|$, by Proposition 2 .

Thus the set of $\alpha$ where $\hat{f}(\alpha) \neq \check{f}(\alpha)$ is at most countable.

d) This follows from parts a) and b).

e) If $\left\{\left(\alpha_{n}, \beta_{n}\right)\right\}$ is a disjoint collection of intervals, then

$$
\begin{aligned}
\sum\left|\check{f}\left(\alpha_{n}\right)-\check{f}\left(\beta_{n}\right)\right| & \leq \sum\left|\check{f}\left(\alpha_{n}\right)-\hat{f}\left(\beta_{n}\right)\right|+\sum\left|\check{f}\left(\beta_{n}\right)-\hat{f}\left(\beta_{n}\right)\right| \\
& =\sum\left|\mu\left(f^{-1}\left(\alpha_{n}, \beta_{n}\right)\right)\right|+\sum\left|\mu\left(f^{-1}\left(\left\{\beta_{n}\right\}\right)\right)\right| \\
& \leq\|\mu\|
\end{aligned}
$$

by a) of Proposition 2. Thus $\check{f}$ is of bounded variation with variation at most $\|\mu\|$.

Since $\check{f}$ is of bounded variation, there is a signed measure $\mu_{f}$ on $\mathbf{R}$ such that $\mu_{f}(\alpha, \beta)=\check{f}(\alpha)-\check{f}\left(\beta^{-}\right)=\check{f}(\alpha)-\hat{f}(\beta)=\mu\left(f^{-1}(\alpha, \beta)\right)$ and $\left\|\mu_{f}\right\|=\left|\mu_{f}\right|(\mathbf{R}) \leq\|\mu\|$. If $O$ is any open set in $\mathbf{R}$, we may write $O$ as a disjoint union of open intervals to see that $\mu_{f}(O)=\mu\left(f^{-1}(O)\right)$. It follows that $\mu_{f}$ is concentrated on sp $f$.

If $f \in C(X)$ and $\varphi \in C(\operatorname{sp} f)$, we let $\varphi^{*} \mu_{f}$ denote the image measure of $\mu_{f}$ under the map $\varphi$. The following lemma simplifies the proof of Proposition 3.2 of [1].

Lemma 4. We have that $\mu_{\varphi \circ f}=\varphi^{*} \mu_{f}$.

Proof. Let $O \subseteq \mathbf{R}$ be open. Then

$$
\begin{aligned}
\mu_{\varphi \circ f}(O) & =\mu\left((\varphi \circ f)^{-1}(O)\right) \\
& =\mu\left(f^{-1}\left(\varphi^{-1}(O)\right)\right) \\
& =\mu_{f}\left(\varphi^{-1}(O)\right) \\
& =\left(\varphi^{*} \mu_{f}\right)(O) .
\end{aligned}
$$

Now we may define the functional $\rho_{\mu}(f)=\int_{\mathbf{R}} i d \mu_{f}$ where $i: \mathbf{R} \rightarrow \mathbf{R}$ is the function $i(x)=x$. Since $\mu_{f}$ is concentrated on sp $f$, we have $\left|\rho_{\mu}(f)\right| \leq \int_{\mathbf{R}}|i| d\left|\mu_{f}\right| \leq$ 
$\|\mu\|\|f\|_{u}$, so $\rho_{\mu}$ is bounded with $\left\|\rho_{\mu}\right\| \leq\|\mu\|$. Also

$$
\begin{aligned}
\rho_{\mu}(\varphi \circ f) & =\int_{\mathbf{R}} i d \mu_{\varphi \circ f} \\
& =\int_{\mathbf{R}} i d \varphi^{*} \mu_{f} \\
& =\int_{\mathbf{R}} \varphi d \mu_{f},
\end{aligned}
$$

so $\rho_{\mu}(\varphi \circ f+\psi \circ f)=\int_{\mathbf{R}} \varphi+\psi d \mu_{f}=\rho_{\mu}(\varphi \circ f)+\rho_{\mu}(\psi \circ f)$. Thus $\rho_{\mu}$ is a quasi-linear functional on $C(X)$.

Theorem 5. The map $\mu \rightarrow \rho_{\mu}$ is an isometric isomorphism of the normed linear space $Q M(X)$ onto $Q L(X)$.

Proof. It is easy to see that this map is linear. We show that it is onto $Q L(X)$.

Suppose $\rho$ is a quasi-linear functional on $C(X)$.

Claim 1: If $U$ is open in $X$ and $\varepsilon>0$, there is a closed $K \subseteq U$ such that if $f \in C(X),\|f\|_{u} \leq 1$, supp $f \subseteq U$, and $f=0$ on $K$, then $|\rho(f)|<\varepsilon$.

Suppose no such $K$ exists for some $\varepsilon>0$. Pick $f_{1} \in C(X)$ such that $\left\|f_{1}\right\|_{u} \leq 1$, supp $f_{1} \subseteq U$, and $\left|\rho\left(f_{1}\right)\right| \geq \varepsilon$. Pick $\left|a_{1}\right|=1$ such that $\rho\left(a_{1} f_{1}\right)=\left|\rho\left(f_{1}\right)\right| \geq \varepsilon$. Now $K_{1}=\operatorname{supp} f_{1}$ fails the conditions of the claim, so there is an $f_{2} \in C(X)$ supported in $U$ such that $\left\|f_{2}\right\| \leq 1, f_{2}=0$ on $K_{1}$, and $\left|\rho\left(f_{2}\right)\right| \geq \varepsilon$. Pick $\left|a_{2}\right|=1$ such that $\rho\left(a_{2} f_{2}\right)=\left|\rho\left(f_{2}\right)\right| \geq \varepsilon$. Since $f_{2}=0$ on the support of $f_{1}$, we have that $\rho\left(a_{1} f_{1}+a_{2} f_{2}\right)=\rho\left(a_{1} f_{1}\right)+\rho\left(a_{2} f_{2}\right) \geq 2 \varepsilon$ and $\left\|a_{1} f_{1}+a_{2} f_{2}\right\|_{u} \leq 1$. Continuing by induction, we may find $f_{n} \in C(X)$ supported in $U$ that vanishes on the support of $a_{1} f_{1}+a_{2} f_{2}+\cdots+a_{n-1} f_{n-1}$ and $\left\|f_{n}\right\| \leq 1$, while $\rho\left(a_{n} f_{n}\right)=\left|\rho\left(f_{n}\right)\right| \geq \varepsilon$. But then $\rho\left(\sum_{i=1}^{n} a_{i} f_{i}\right) \geq n \varepsilon$, which violates the boundedness of $\rho$ for large $n$.

Claim 2: For $U$ open, $\lim _{f \prec U} \rho(f)$ exists where the $f$ are ordered pointwise.

We show that this net is a Cauchy net. In fact, let $\varepsilon>0$ and let $K \subseteq U$ be the closed set of Claim 1. Let $f$ be any function such that $f=1$ on $K$ and $f \prec U$. If $f \leq g, h \prec U$, then pick $k$ with $k=1$ on supp $g \cup \operatorname{supp} h$, and $k \prec U$. Then $g-k$ and $h-k$ vanish on $K$, so we have that $|\rho(g)-\rho(h)| \leq|\rho(g)-\rho(k)|+|\rho(h)-\rho(k)|=$ $|\rho(g-k)|+|\rho(h-k)| \leq 2 \varepsilon$.

Define $\mu(U)=\lim _{f \prec U} \rho(f)$ for $U$ open in $X$.

Claim 3: $\mu$ is a signed quasi-measure on $X$.

Easily, $\mu(U \cup V)=\mu(U)+\mu(V)$ if $U$ and $V$ are disjoint. Also $\mu(\emptyset)=0$. Notice also that $\mu(X)=\rho(1)$. We next show property b) after the definition of a signed quasi-measure.

Suppose that $U \cup V=X$. Pick $C \subseteq U$ and $K \subseteq V$, closed such that $C \cup K=X$. Pick $f_{0} \prec U, g_{0} \prec V$ with $f_{0}=1$ on $C, g_{0}=1$ on $K$, and such that $f_{0} \leq f \prec$ $U$ implies $|\rho(f)-\mu(U)|<\varepsilon$ and $g_{0} \leq g \prec V$ implies $|\rho(g)-\mu(V)|<\epsilon$. Let $h_{0} \prec U \cap V$ with $h_{0}^{2}=1$ on $C \cap K$ and such that $h_{0}^{2} \leq h \prec U \cap V$ implies that $|\rho(h)-\mu(U \cap V)|<\varepsilon$. Now, set $f=\max \left\{f_{0}, h_{0}\right\}$ and $g=\max \left\{g_{0}, h_{0}\right\}$. Then $f \prec U, g \prec V$, and $f g \prec U \cap V$, so $|\rho(f)-\mu(U)|<\varepsilon,|\rho(g)-\mu(V)|<\varepsilon$ and $|\rho(f g)-\mu(U \cap V)|<\varepsilon$. Also, since $f=1$ on $C$ and $g=1$ on $K$, and $C \cup K=X$, we have that $(1-f)(1-g)=0$, so $\rho((1-f)+(1-g))=\rho(1-f)+\rho(1-g)$ and 
$\rho(f+g)=\rho(1+f g)$. This gives that $\rho(f)+\rho(g)=\rho(f+g)=\rho(1)+\rho(f g)$, which shows that $|\mu(U)+\mu(V)-\mu(X)-\mu(U \cap V)|<3 \varepsilon$.

Now suppose that $\left\{U_{n}\right\}_{n=1}^{N}$ is a finite, disjoint collection of open sets. Let $\varepsilon>0$ be given and choose $f_{n} \prec U_{n}$ such that $\left|\rho\left(f_{n}\right)-\mu\left(U_{n}\right)\right|<\varepsilon$. Now choose $\left|a_{n}\right|=1$ such that $\left|\rho\left(f_{n}\right)\right|=\rho\left(a_{n} f_{n}\right)$. Then $\left|\rho\left(\sum a_{n} f_{n}\right)\right| \leq\|\rho\|$, so $\sum\left|\mu\left(U_{n}\right)\right| \leq\|\rho\|+N \varepsilon$. Now let $\varepsilon \rightarrow 0$.

Finally, if $U$ an open set and $\varepsilon>0$ are given, choose $K \subseteq U$ as in Claim 1, and argue as in the previous paragraph to show that if $\left\{U_{n}\right\}$ are disjoint and open with $U_{n} \subseteq U \backslash K$, then $\sum\left|\mu\left(U_{n}\right)\right|<\varepsilon$. In particular, if $V \subseteq U \backslash K$ is open, $|\mu(V)|<\varepsilon$.

Notice that $\|\mu\| \leq\|\rho\|$.

Claim 4: We have that $\rho=\rho_{\mu}$.

For each $f \in C(X)$, the map $\varphi \rightarrow \rho(\varphi \circ f)$ is bounded and linear on $C($ sp $f)$. Thus, there is a signed measure $\nu_{f}$ on sp $f$ such that

$$
\rho(\varphi \circ f)=\int_{\mathbf{R}} \varphi d \nu_{f}
$$

for all $\varphi \in C(\operatorname{sp} f)$. Since $\rho(f)=\int_{\mathbf{R}} i d \nu_{f}$, we need only show that $\nu_{f}=\mu_{f}$ for each $f \in C(X)$. Notice that both measures are measures on $\mathbf{R}$.

Suppose that $O$ is an open set in $\mathbf{R}$. Pick closed sets $C_{n} \subseteq O$ such that $C_{n} \subseteq$ $\operatorname{int}\left(C_{n+1}\right)$ and $O=\bigcup C_{n}$. Choose $\varphi_{n} \prec O$ such that $\varphi_{n}=1$ on $C_{n}$. Since $X$ is compact, the sequence $\varphi_{n} \circ f$ is cofinal in the collection of functions $g$ such that $g \prec f^{-1}(O)$. Thus

$$
\begin{aligned}
\nu_{f}(O) & =\lim _{n \rightarrow \infty} \int \varphi_{n} d \nu_{f} \\
& =\lim _{n \rightarrow \infty} \rho\left(\varphi_{n} \circ f\right) \\
& =\mu\left(f^{-1}(O)\right) \\
& =\mu_{f}(O)
\end{aligned}
$$

giving the required equality of measures. Thus $\|\mu\| \leq\|\rho\|=\left\|\rho_{\mu}\right\| \leq\|\mu\|$.

This shows that the map $\mu \rightarrow \rho_{\mu}$ is onto $Q L(X)$, and in fact, that any $\rho \in Q L(X)$ is the image of some $\mu \in Q M(X)$ of the same norm. If we show that our map is one-to-one, we will be finished.

Assume $\rho_{\mu}=0$. Then $\mu_{f}=0$ for all $f \in C(X)$. Thus $\check{f}(\alpha)=\mu_{f}(\alpha,+\infty)=0$ for all $\alpha \in \mathbf{R}$. If, now, $U \subseteq X$ is open and $\varepsilon>0$, pick $K \subseteq U$ as in part (iv) of the definition of a signed quasi-measure. Choose any $f \in C(X)$ with $K \prec f \prec U$. Then

$$
\begin{aligned}
|\mu(U)| & \leq|\mu(U)-\mu(K)|+\left|\check{f}\left(\frac{1}{2}\right)-\mu(K)\right|+\left|\check{f}\left(\frac{1}{2}\right)\right| \\
& =|\mu(U \backslash K)|+\left|\mu\left(\left(f^{-1}\left(\frac{1}{2},+\infty\right)\right) \backslash K\right)\right|+\left|\check{f}\left(\frac{1}{2}\right)\right| \\
& \leq 2 \varepsilon .
\end{aligned}
$$

Thus $\mu(U)=0$ for all open sets, so $\mu=0$.

It should be noted that in Claim 2 we actually showed a stronger form of regularity. If $U$ is open and $\varepsilon>0$, then there is a closed set $K \subseteq U$ with $|\mu|(U \backslash K)<\varepsilon$. Thus $|\mu|$ obeys part (iv) of the definition of a quasi-measure. 
There is yet another representation of $Q L(X)$ that is sometimes useful. For each $f \in C(X)$, let $M(\operatorname{sp} f)$ denote the collection of regular Borel measures on the compact set sp $f$ with the usual measure norm. Define $P M(X)$ to be

$$
\left\{\left(\nu_{f}\right) \in \prod_{f \in C(X)} M(\operatorname{sp} f): \nu_{\varphi \circ f}=\varphi^{*} \nu_{f} \text { for } \varphi \in C(\operatorname{sp} f) \text { and } \sup \left\|\nu_{f}\right\|<\infty\right\} .
$$

Define a norm on $P M(X)$ by $\left\|\left(\nu_{f}\right)\right\|=\sup \left\|\nu_{f}\right\|$. Then it is easy to see that $P M(X)$ is a Banach space since this is true for each $M(\operatorname{sp} f)$.

If $\mu$ is a signed quasi-measure, then the collection $\left(\mu_{f}\right)$ in the definition of $\rho_{\mu}$ is an element of $P M(X)$ with $\left\|\left(\mu_{f}\right)\right\| \leq\|\mu\|$. The induced map from $Q M(X)$ to $P M(X)$ is evidently linear.

On the other hand, if $\left(\nu_{f}\right) \in P M(X)$, we may define $\rho(f)=\int_{\mathbf{R}} i d \nu_{f}$. Then the argument just before the statement of the theorem shows that $\rho \in Q L(X)$ with $\|\rho\| \leq\left\|\left(\nu_{f}\right)\right\|$. This, with the last paragraph shows that $P M(X)$ is isometrically isomorphic to both $Q M(X)$ and $Q L(X)$.

In particular $Q M(X)$ is a Banach space. We can make it an ordered Banach space by taking the positive cone to be the collection of positive quasi-measures. The norm on this space is additive on the positive cone, but $Q M(X)$ does not have to be a lattice. Thus, $Q M(X)$ need not be an $L$-space. For example, in [3], Aarnes finds positive $\{0,1\}$-valued quasi-measures $\mu_{1}, \mu_{2}, \mu_{3}, \mu_{4}$ with $\mu_{1}+\mu_{3}=\mu_{2}+\mu_{4}$. Since $\{0,1\}$-valued quasi-measures are extremal, there will then be no supremum of $\left\{\mu_{1}, \mu_{2}\right\}$.

For future convenience, we define the notation $\langle\mu, f\rangle=\left\langle\rho_{\mu}, f\right\rangle=\rho_{\mu}(f)$.

Another aspect of the failure of the lattice property is that the set function $|\mu|$ need not have an extension to a positive quasi-measure on $X$. An example of this is given next.

Example. Let $X=[0,1] \times[0,1]$. In [2] and [6], it is shown how to construct the so-called three point quasi-measures. This is done as follows. A subset $A$ of $X$ is said to be solid if both $A$ and $X \backslash A$ are connected. If $C=\left\{x_{1}, x_{2}, x_{3}\right\}$ is a set with three elements, we define $\mu_{C}$ on solid sets by

$$
\mu_{C}(A)= \begin{cases}0 & \text { if } \operatorname{card}(A \cap C) \leq 1 \\ 1 & \text { if } \operatorname{card}(A \cap C) \geq 2\end{cases}
$$

There is then a unique extension of $\mu_{C}$ to a $\{0,1\}$-valued quasi-measure on $X$.

Now let $x_{1}=(0,0), x_{2}=(1,0), x_{3}=(1,1)$, and $x_{4}=(0,1)$. Let $C=\left\{x_{1}, x_{2}, x_{3}\right\}$, $D=\left\{x_{2}, x_{3}, x_{4}\right\}$, and $\mu=\mu_{C}-\mu_{D}$. If we let $U_{1}=[0,1] \times\left[0, \frac{1}{2}\right)$ and $U_{2}=$ $[0,1] \times\left(\frac{1}{2}, 1\right]$, we see that $2=\left|\mu\left(U_{1}\right)\right|+\left|\mu\left(U_{2}\right)\right| \leq|\mu|(X)=\|\mu\| \leq 2$. Hence, $|\mu|(X)=2$. We show that $|\mu|$ cannot be extended to closed sets in such a way that it is a positive quasi-measure. In particular, (ii) does not hold in the definition of a quasi-measure.

Assume such an extension exists. Let $V_{1}=\left[0, \frac{3}{4}\right) \times[0,1]$ and $V_{2}=\left(\frac{1}{4}, 1\right] \times[0,1]$. Write $K_{1}=X \backslash V_{1}$ and $K_{2}=X \backslash V_{2}$. Since $\mu_{C}\left(V_{1}\right)=\mu_{D}\left(V_{1}\right)=0$, we see that $|\mu|\left(V_{1}\right)=0=|\mu|\left(V_{1} \cap V_{2}\right)$. Hence $|\mu|\left(K_{1}\right)=|\mu|\left(K_{1} \cup K_{2}\right)=2$. Since $K_{1}$ and $K_{2}$ are disjoint, we must have that $|\mu|\left(K_{2}\right)=0$, in other words that $|\mu|\left(V_{2}\right)=2$. However this is not the case. In fact, $|\mu|\left(V_{2}\right)=0$.

To see this we show that if $U \subseteq V_{2}$ is open, then $\mu(U)=0$; that is $\mu_{C}(U)=$ $\mu_{D}(U)$. Using symmetry and the fact that both $\mu_{C}$ and $\mu_{D}$ take on only 0 and 
1 as values, we only show that $\mu_{C}(U)=0$ implies $\mu_{D}(U)=0$. Furthermore, by considering components, it is enough to show this for connected open sets.

Suppose, then, that $U$ is connected and let $K$ be any connected closed subset of $U$. Let $\hat{K}$ be the solid hull of $K$ as a subset of $V_{2}$ as in [2]. Then $\hat{K}$ is a solid closed set in $V_{2}$ containing $K$. If $\mu_{C}(\hat{K})=0$, then $\left\{x_{2}, x_{3}\right\} \cap \hat{K}$ has at most one element, so $\mu_{D}(K) \leq \mu_{D}(\hat{K})=0$. On the other hand, if $\mu_{C}(\hat{K})=1$, there is some interior component $W$ of $X \backslash K$ with $\mu_{C}(W)=1$. This follows since $\mu_{C}(K)=0$. Since $W \subseteq \hat{K} \subseteq V_{2}$, we have that $x_{2}, x_{3} \in W$. Since $W$ is solid, we then have that $\mu_{D}(W)=1$, so $\mu_{D}(K) \leq \mu_{D}(X \backslash W)=0$. Finally, $\mu_{D}(U)$ is the supremum of $\mu_{D}(K)$ as above, so $\mu_{D}(U)=0$.

It would be nice to know that every signed quasi-measure is the difference of two positive quasi-measures. However, the failure of the lattice property and the fact that $|\mu|$ need not be a quasi-measure brings this into question. At this point the issue remains open.

We now turn to another topology on $Q M(X)$ that is very useful.

Definition 6. The weak-* topology on $Q M(X)$ is the weakest topology making each map $\mu \rightarrow\langle\mu, f\rangle$ continuous where $f$ ranges over $C(X)$.

Since each such map is linear and the collection of these maps separates points of $Q M(X)$, we obtain a locally convex topology on $Q M(X)$ where a net $\mu_{\alpha}$ converges to $\mu$ if and only if $\left\langle\mu_{\alpha}, f\right\rangle$ converges to $\langle\mu, f\rangle$ for every $f \in C(X)$. This topology has been studied on the space of positive quasi-measures in [3].

Proposition 7. Let $\mu_{\alpha}$ be a net in $Q M(X)$ and $\mu \in Q M(X)$. Let $\left(\mu_{\alpha, f}\right)$ and $\left(\mu_{f}\right)$ be the corresponding elements of $P M(X)$. Then $\mu_{\alpha}$ converges to $\mu$ in the weak-* topology if and only if $\mu_{\alpha, f}$ converges to $\mu_{f}$ in the weak-* topology of $M(\mathrm{sp} f)$ for each $f \in C(X)$. Thus, the unit ball in $Q M(X)$ is weak-* compact.

Proof. If $\mu_{\alpha, f}$ converges to $\mu_{f}$ for each $f \in C(X)$, then $\left\langle\mu_{\alpha}, f\right\rangle=\int_{\mathbf{R}} i d \mu_{\alpha, f}$ converges to $\int_{\mathbf{R}} i d \mu_{f}=\langle\mu, f\rangle$.

Conversely, if $\mu_{\alpha}$ converges to $\mu$ weak-*, then for each $\varphi \in C(\operatorname{sp} f)$, we have

$$
\begin{aligned}
\lim _{\alpha} \int_{\mathbf{R}} \varphi d \mu_{\alpha, f} & =\lim _{\alpha}\left\langle\mu_{\alpha}, \varphi \circ f\right\rangle \\
& =\langle\mu, \varphi \circ f\rangle \\
& =\int_{\mathbf{R}} \varphi d \mu_{f} .
\end{aligned}
$$

Thus $\mu_{\alpha, f}$ converges to $\mu_{f}$ in the weak-* topology.

Since the map $\mu_{f} \rightarrow \varphi^{*} \mu_{f}$ is weak-* continuous, the compactness of the unit ball of $Q M(X)$ follows from the compactness of the unit balls of $M(\operatorname{sp~} f)$.

Definition 8. Let $X$ and $Y$ be compact Hausdorff spaces. A quasi-linear map from $C(X)$ to $C(Y)$ is a map, $T$, which is linear on each singly generated subalgebra of $C(X)$ and which is bounded in the sense that there is an $M<\infty$ with $\|T(f)\| \leq$ $M\|f\|$. If, in addition, $T$ is multiplicative on each singly generated subalgebra, we say that $T$ is a quasi-homomorphism.

For example, let $\rho$ be a positive quasi-linear functional on $X$ and let $Y$ be any compact Hausdorff space. Define $T_{\rho}: C(X \times Y) \rightarrow C(Y)$ by $T_{\rho}(f)(y)=\rho\left(f^{y}\right)$ where $f^{y}(x)=f(x, y)$. It is noted in [5] that $T_{\rho}$ is a quasi-linear map. If $\eta: C(Y) \rightarrow \mathbf{R}$ 
is an additional quasi-linear functional, it is also noted there that the composition of $T_{\rho}$ and $\eta$ need not be quasi-linear.

Proposition 9. There is a one to one correspondence between quasi-linear maps from $C(X)$ to $C(Y)$ and norm-bounded functions $Y \rightarrow Q M(X)$ which are weak-* continuous. Specifically, if $y \rightarrow \mu_{y}$ is a bounded, weak-* continuous map of $Y$ into $Q M(X)$, the corresponding quasi-linear map is $T(f)(y)=\left\langle\mu_{y}, f\right\rangle$.

The proof of this is evident. For comparison, there is a similar correspondence between the quasi-homomorphisms from $C(X)$ to $C(Y)$ and weak-* continuous functions $Y \rightarrow X^{*}$ where $X^{*}$ is the collection of $\{0,1\}$-quasi-measures on $X$. See [4] for details.

Proposition 10. Let $T: C(X) \rightarrow C(Y)$ be a quasi-homomorphism and $S: C(Y)$ $\rightarrow C(Z)$ be a quasi-linear map. Then the composition $S \circ T: C(X) \rightarrow C(Z)$ is a quasi-linear map. If $y \rightarrow \mu_{y}$ is the map from $Y$ to $X^{*}$ corresponding to $T, z \rightarrow \nu_{z}$ the map from $Z$ into $Q M(Y)$ corresponding to $S$, and $z \rightarrow \omega_{z}$ the map from $Z$ to $Q M(X)$ corresponding to $S \circ T$, then for $U$ open in $X$,

$$
\omega_{z}(U)=\nu_{z}\left(\left\{y \in Y: \mu_{y}(U)=1\right\}\right) .
$$

Proof. That the composition is quasi-linear is evident.

Let $U \subseteq X$ be open and $\varepsilon>0$. Let $W=\left\{y: \mu_{y}(U)=1\right\}$.

Claim: For each $y \in W$ there is a compact $K_{y} \subseteq U$ and open set $y \in V_{y}$ such that $y^{\prime} \in V_{y}$ implies $\mu_{y^{\prime}}\left(K_{y}\right)=1$.

Since $\mu_{y}(U)=1$, there is a compact $K_{1} \subseteq U$ with $\mu_{y}\left(K_{1}\right)=1$. Let $f \in C(X)$ with $K_{1} \prec f \prec U$. Then $\left\langle\mu_{y}, f\right\rangle=1$, so by weak-* continuity, there is an open set $y \in V$ such that $y^{\prime} \in V$ implies that $\left\langle\mu_{y^{\prime}}, f\right\rangle>\frac{2}{3}$. Let $K_{y}=\left\{x: f(x) \geq \frac{1}{3}\right\}$. Since each $\mu_{y^{\prime}}$ is a $\{0,1\}$-quasi-measure, $\mu_{y^{\prime}}\left(K_{y}\right)=1$ for $y^{\prime} \in V$.

In particular, we see that $W$ is open in $Y$. This also shows that if $A \subseteq X$ is closed then $\left\{y: \mu_{y}(A)=1\right\}$ is closed in $Y$. Now we can find compact sets $K \subseteq U$ and $C \subseteq W$ such that $K \prec f \prec U$ implies $\left|\left\langle\omega_{z}, f\right\rangle-\omega_{z}(U)\right|<\varepsilon$ and $C \prec g \prec W$ implies $\left|\left\langle\nu_{z}, g\right\rangle-\nu_{z}(W)\right|<\varepsilon$.

For each $y \in C$, pick $V_{y}$ and $K_{y}$ as in the claim. Choose finitely many $V_{y}$ to cover $C$, say $V_{1}, \cdots, V_{n}$. Let $K_{1}, \cdots, K_{n}$ be the corresponding $K_{y}$.

Set $L=K \cup K_{1} \cup \cdots \cup K_{n}$ and find $L \prec f \prec U$. Recall $T(f)(y)=\left\langle\mu_{y}, f\right\rangle$ for $y \in Y$. If $y \in C$, say $y \in V_{j}, 1 \leq \mu_{y}\left(K_{j}\right) \leq \mu_{y}(L) \leq\left\langle\mu_{y}, f\right\rangle \leq \mu_{y}(U) \leq 1$, so $C \prec T(f)$. Also, if $F$ is the support of $f$, then $E=\left\{y: \mu_{y}(F)=1\right\}$ is closed and contained in $W$. If $y \notin E, \mu_{y}(F)=0$, so $\left\langle\mu_{y}, f\right\rangle=0$. Thus, the support of $T(f)$ is contained in $E \subseteq W$. Hence $C \prec T(f) \prec W$.

Thus, $\left|\omega_{z}(U)-\left\langle\omega_{z}, f\right\rangle\right|<\varepsilon$ and $\left|\nu_{z}(W)-\left\langle\nu_{z}, T(f)\right\rangle\right|<\varepsilon$. However, $\left\langle\omega_{z}, f\right\rangle=$ $S \circ T(f)(z)=\left\langle\nu_{z}, T(f)\right\rangle$. Hence $\left|\omega_{z}(U)-\nu_{z}(W)\right|<2 \varepsilon$. Now let $\varepsilon$ go to 0 .

Another interpretation of this result may be obtained by noting that a quasihomomorphism $T: C(X) \rightarrow C(Y)$ induces an image transformation $q: \mathcal{A}(X) \rightarrow$ $\mathcal{A}(Y)$ (see [4] for details). In particular, for $A \in \mathcal{A}(X)$, we have $q(A)=\left\{y: \mu_{y}(A)=\right.$ $1\}$. For $\nu \in Q M(Y)$, we can then define $q^{*} \nu \in Q M(X)$ by $q^{*} \nu(A)=\nu(q(A))$. The above proposition then states that $\omega_{z}=q^{*} \nu_{z}$ for $z \in Z$.

I would like to thank Johan Aarnes and Robert Wheeler for their encouragement to pursue this paper. 


\section{REFERENCES}

[1] Aarnes, Johan, Quasi-states and Quasi-measures, Advances in Mathematics 86 (1991) 41-67. MR 92d: 46152

[2] Aarnes, Johan, Construction of Non Subadditive Measures and Discretization of Borel Measures, Fund. Math. 147 (1995) 213-237. MR 96k:28022

[3] Aarnes, Johan, Pure quasi-states and extremal quasi-measures, Mathematische Annalen 295 (1993) 575-588. MR 94e:46096

[4] Aarnes, Johan, Image Transformations, Attractors and Invariant Non-subadditive Measures, preprint.

[5] Grubb, D. J., Products of Quasi-measures, Proc. Amer. Math. Soc. 124 (1996) 2161-2166. MR 96i: 28016

[6] Knudsen, Finn, Topology and the Construction of Extreme Quasi-measures, Advances in Mathematics 120 (1996) 302-321. CMP 96:14

Department of Mathematics, Northern Illinois University, DeKalb, Illinois 60115

E-mail address: grubb@math.niu.edu 